\title{
Study on Semi-Parametric Statistical Model of Safety Monitoring of Cracks in Concrete Dams
}

\author{
Chongshi Gu, ${ }^{1,2}$ Dong Qin, ${ }^{1,2}$ Zhanchao Li, ${ }^{1,3}$ and Xueqin Zheng ${ }^{1,2}$ \\ ${ }^{1}$ State Key Laboratory of Hydrology-Water Resources and Hydraulic Engineering, Hohai University, Jiangsu Nanjing 210098, China \\ ${ }^{2}$ College of Water Conservancy and Hydropower Engineering, Hohai University, Jiangsu Nanjing 210098, China \\ ${ }^{3}$ College of Water Resources and Architectural Engineering, Northwest A\&F University, Shanxi Yangling 712100, China
}

Correspondence should be addressed to Dong Qin; qddamsafe@163.com

Received 17 April 2013; Revised 25 June 2013; Accepted 9 July 2013

Academic Editor: Vishal Bhatnagar

Copyright (C) 2013 Chongshi Gu et al. This is an open access article distributed under the Creative Commons Attribution License, which permits unrestricted use, distribution, and reproduction in any medium, provided the original work is properly cited.

\begin{abstract}
Cracks are one of the hidden dangers in concrete dams. The study on safety monitoring models of concrete dam cracks has always been difficult. Using the parametric statistical model of safety monitoring of cracks in concrete dams, with the help of the semiparametric statistical theory, and considering the abnormal behaviors of these cracks, the semi-parametric statistical model of safety monitoring of concrete dam cracks is established to overcome the limitation of the parametric model in expressing the objective model. Previous projects show that the semi-parametric statistical model has a stronger fitting effect and has a better explanation for cracks in concrete dams than the parametric statistical model. However, when used for forecast, the forecast capability of the semiparametric statistical model is equivalent to that of the parametric statistical model. The modeling of the semi-parametric statistical model is simple, has a reasonable principle, and has a strong practicality, with a good application prospect in the actual project.
\end{abstract}

\section{Introduction}

In an investigative report on the state of dams, the International Committee on Large Dams points out that the majority of concrete dams in various countries have cracks, in which a total of 243 dams have collapsed due to these cracks [1]. Cracks are one of the hidden dangers in concrete dams, which are greatly dangerous to their safety. Serious cracks will deteriorate the strength and stability of a dam's body structure, damage its integrity and antipermeability, accelerate the carbonization and corrosion of concrete, and endanger its safe operation [1]. Around the world, there have been many dams whose operations were affected due to cracks, such as the solid gravity dam in Dworshak in America [2]. It has a height of $219 \mathrm{~m}$, and during its construction, there were no serious structural cracks, but after operating for several years, cracks were found on nine of the dam's sections, in which the crack on section 35 was the most serious, with a depth of $50 \mathrm{~m}$, a width of $2.5 \mathrm{~mm}$, and a water percolating capacity of $483 \mathrm{~L} / \mathrm{s}$. In addition, the Zillergrund arch dam in Austria, the Zeuzier arch dam in Switzerland, the Revelstoke solid gravity dam in Canada, the Sayano-Shushenskaya gravity arch dam in Russia, the Koyna gravity dam in India, the Pacoima arc dam in America, and the Sefid Rud buttress dam in Iran were also seriously affected by cracks.

Up to now, the key technologies of concrete dams are still very difficult subjects to research on, with aspects such as evolution law, development trends, and abnormal instability to consider. Crack aperture is an important parameter for determining a crack's development, evolution, and how it would deform a dam's structure. It is significant to establish an appropriate mathematical model in order to accurately determine the aperture of cracks and the changes on their pattern, so as to judge whether these cracks have rendered the dam unstable and to monitor the changes in the concrete dam's structure to prevent accidents from occurring. To this end, $\mathrm{Wu}$ and $\mathrm{Gu}$ [3] proposed the parametric statistical model of safety monitoring of cracks in concrete dams, with hydraulic pressure, temperature, and timeliness as its main factors, and this method has been widely applied in several projects. However, in affecting a crack's aperture, hydraulic pressure and temperature sometimes are not mutually independent variables. There exists a nonlinear correlation between them, and it is difficult for the parametric statistical model to 
separate various components, and the relevant components of such nonlinearity are partially transferred to the residual error. It is thought in this statistical model that flexible crack deformations caused by hydraulic pressure and temperature loads are recoverable, while the crack deformations caused by time are unrecoverable. However, due to hydraulic pressure and temperature, the stress field at the tip of a crack has a singularity, making further irreversible damages. This deformation is not part of the hydraulic pressure and temperature components of this model, but it is part of the residual error component [4]. By further studying the abnormal behaviors of cracks in concrete dams [5-7], it is found that, when such abnormal behavior is observed, mutation occurs to the crack's aperture sequence. In this model, hydraulic pressure, temperature, and timeliness components are the continuous functions of corresponding factors. The combination of hydraulic pressure, temperature, timeliness, and crack aperture components generate a residual error.

Based on the problems stated previously, many scholars have modified and improved the parametric statistical model of safety monitoring of cracks in concrete dams. Reference [8] regarded a concrete dam as an uncertain system and adopted the approximate reasoning model and information distribution method to establish the uncertain model of concrete dam crack aperture. Reference [2] introduced two factors, namely, crack depth and crack initial aperture, to improve the parametric statistical model. Reference [9] studied the influence of crack length on the crack aperture and established the crack abnormality monitoring model in combination with the crack safety monitoring theory and its abnormality diagnosis method. Reference [10] studied the general principle of the statistical and chaotic hybrid forecast model and proposed that such model be used for measuring concrete dam crack aperture. Reference [11] applied the RS rough set theory to simplify the property and sample set of crack monitoring information and the BP neural network for model training to simplify the sample set and proposed a crack aperture monitoring model based on such RS-BP coupling. Reference [12] established a hybrid model of safety monitoring of concrete dam aperture by analyzing the relation between land change and crack aperture. These studies show that the existing models are still based on the parametric statistical model these models do not solve the relevant and nonlinear problems that are posed by the such statistical model, and they are also unable to measure the abnormality of a crack's behavior.

As a result, considering the problems in the parametric statistical model and by introducing the nonparametric component of concrete dam cracks to express the influence in crack openings, this paper establishes the semiparametric statistical model of safety monitoring of cracks in concrete dams to overcome the limitations of the parametric model.

\section{Construction of Semiparametric Statistical Model of Safety Monitoring of Cracks in Concrete Dams}

In the semiparametric model, the function model is divided into three parts, namely, a finite-dimensional parameter component (trend component), a nonparametric component (abnormal component) belonging to infinite-dimensional function space, and a random error component. The abnormal component represents the unknown component (such as a system error or model deviation) of a functional relationship, and the trend component describes the known ingredients of function in the observed data. For the semiparametric model, Green and Silverman [13] proposed smoothing spline estimation methods, Eubank et al. [14] studied the trigonometric series estimation method, Ivanov and Leonenko [15] studied large sample properties of errors' semiparametric models under long-range dependence, Shi and Chai [16] proposed the basic theory and estimation methods of the semiparametric regression model with the smoothing least squares local polynomial, Zen [17] studied statistical diagnostics with the semiparametric generalized linear model, $\mathrm{Hu}[18]$ studied the strong consistency of wavelet estimators in martingale difference using the semiparametric regression model, and Ruppert et al. [19] systematically studied the semiparametric theory and methods. For the theories and methods in kernel estimation, S. Wang and C. Y. Wang [20] studied the semiparametric model kernel estimation when the covariate is lost or when measurement errors occur, Hong [21] studied the data-driven kernel density estimation in the semiparametric regression model, Robinson and Henry [22] studied high-level nuclear semiparametric estimation under long-range dependence, Manzan and Zerom [23] studied kernel density estimation with partial linear additive model, Hjort and Walker [24] studied the kernel density estimation in optimal window width, Hagmann and Scaillet [25] studied bias correction of asymmetric kernel density estimation, and Song [26] studied the moderate bias of deconvolution kernel density estimation when the measurement errors are smooth and are stationarily distributed. Many studies show that, while the semiparametric model is used in solving the complex relationships between estimated parameters and observations, the relative parametric or nonparametric model has obvious advantages which has been widely used in industry, agriculture, economics, medicine, finance, and other fields.

The application of the semiparametric regression model in the field of hydraulic structures safety monitoring is a new research tool and method. Currently, there are only a few published studies on this model. Xu et al. [27] took the abstracted principal components as semiparametric regression parameter components, took the remaining ingredients and model error as unknown nonparametric components using principal component analysis to compensate for the principal component regression, and established the dam deformation monitoring mixed regression model in an attempt to apply the semiparametric regression model into the field of hydraulic structures safety monitoring. This paper combines the research results of existing concrete dam safety monitoring models, focuses on fractured abnormality properties, creates the semiparametric monitoring model, and tests the model's validity.

2.1. Semiparametric Regression Statistical Model. In concrete dam cracks, the influence factors are relatively complex. There have been more analyses and studies on the generation 
and development mechanism of concrete dam cracks, and researchers have posted the following findings [28]: (1) it is thought that the existence of a crack during the construction period is an important reason for the expansion of cracks at a later stage; (2) it is thought that the unfavorable load combination during the operation of the dam is an important factor for the generation and development of cracks; and (3) it is thought that the cracks are influenced by concrete rheology, crack side plastic aperture, and seepage effects, which are important factors for the generation and expansion of cracks. Therefore, the factors influencing the crack's aperture can boil down to the external load (like hydraulic pressure and temperature) over time. Meanwhile, when the material near the top end of the crack reaches the maximum allowable strain, the crack will be expanded. The modification in the plastic zone is considered, and the formula for the crack aperture is described below [29]:

$$
K_{0}=\frac{4 \sigma}{E} \sqrt{\left(a+r_{p}^{*}\right)^{2}-x^{2}},
$$

where $\sigma$ is the strain near the top of the crack, $E$ is the elasticity modulus, $a$ is half of the crack's length, $r_{p}^{*}$ is the radius of the plastic zone, and $x$ is the distance to the top of the crack.

The previous formula states that a concrete dam's aperture is related to $\sigma$ and is the joint result of external load and crack side plastic deformation. Thus, the statistical model of crack aperture can be expressed as

$$
K=K(H)+K(T)+K(\theta)+\varepsilon,
$$

where $K$ is the crack aperture, $K(H)$ is the component of crack aperture caused by hydraulic pressure factor, $K(T)$ is the component of crack aperture caused by temperature factor, $K(\theta)$ is the component caused by timeliness factor, and $\varepsilon$ is the random error term. The principle for the selection of each component in the formula is as follows.

In the regression model, if the factors influencing the explained variable $Y$ are divided into two parts, that is, $X_{1}, \ldots, X_{p}$ and $t_{1}, \ldots, t_{q}$, according to experience and historical data, it can be thought that $X_{1}, \ldots, X_{p}$ are the main parts, and $Y$ is linear with $X_{1}, \ldots, X_{p}$. While $t_{1}, \ldots, t_{q}$ is a certain disturbance factor, the relation between it and $Y$ is completely unknown, but as its influence is systematical, it is not suitable to include it in the accidental error term. At this moment, if the nonparametric regression model is used for treatment, too much information will be lost. If the linear model is used for treatment, the terms representing error will surely contain systematical components and will no longer conform to the random characteristics of accidental error, thus will make the fitting effect very poor. In this paper, the semiparametric regression model of concrete dam crack openings' monitoring sequence is built.

The general form of the semiparametric model is

$$
L=f(X)+g(Z)+\varepsilon
$$

where $L$ is the response variable, $X$ and $Z$ are the relevant concomitant variables, $\varepsilon$ is the error term, $f(\cdot)$ is the clear relation between the observation value and some parameters, which is the parametric component of the model, and $g(\cdot)$ is the part without a clear function relation between the observation value and the parameter, which is called the nonparametric component of the model.

The semiparametric model is a widely applied statistical model which is deeply focused on the semiparametric regression model. It includes both the parametric regression and the nonparametric regression models. In the semiparametric model, the linear semiparametric model is a kind of a time sequence model that has been used frequently over the recent years. It does not only include a parametric part, but also a nonparametric part. The linear part controls the overall trend of the explained variable, which is applicable for the extension forecast. Meanwhile, the nonparametric part makes a partial adjustment to the explained variable, which makes the model fit better to the sample observation model. Thus, the linear semiparametric model can be expressed as

$$
Y=X B+g(t)+\varepsilon,
$$

where $B=\left(B_{1}, \ldots, B_{p}\right)^{T}, p \geq 1$ is the unknown parameter, $Y=\left(Y_{1}, \ldots, Y_{n}\right)^{T}, X=\left(X_{1}, \ldots, X_{n}\right)^{T}, g=\left(g\left(t_{1}\right), \ldots, g\left(t_{n}\right)\right)$, and $g\left(t_{i}\right)$ are the unknown functions defined between $[0,1]$, $\varepsilon=\left(\varepsilon_{1}, \ldots, \varepsilon_{n}\right)$ is the random error vector of independent identical distribution, and $E\left(\varepsilon_{i}\right)=0, E\left(\varepsilon_{i}^{2}\right)<\infty, t_{i} \in[0,1]$, $0 \leq t_{1}<t_{2}<\cdots<t_{n} \leq 1, t_{i}$, and $\varepsilon_{i}$ are the mutually independent parameters.

The estimation problem of the semiparametric model is a point estimation problem of Euclidean space with infinitedimension nuisance shape parameter, and the estimation methods that are more focused on are the least-square method kernel estimation, spline estimation, partitional polynomial estimation, and trigonometric series estimation [30]. These methods are adopted in this paper to solve the parameter estimation problem of the semiparametric model.

Assuming the probability density kernel function as $K(\cdot)$ and $h>0 R$ on, for the selected point $t_{k}$, the kernel function $W_{i}\left(t_{k}\right)$ is computed as

$$
W_{i}\left(t_{k}\right)=\frac{K\left(\left(t_{k}-t_{i}\right) / h\right)}{\sum_{j=1}^{n} K\left(\left(t_{k}-t_{j}\right) / h\right)}, \quad i, j, k=1, \ldots, n .
$$

Assume that $B$ is known, the nonparametric kernel estimation of $g\left(t_{k}\right)$ is made based on $\left\{Y_{i}-X_{i} B, t_{i}\right\}_{i-1}^{n}$ :

$$
\widehat{g}\left(t_{k}, B\right)=\sum_{i=1}^{n} W_{i}\left(t_{k}\right)\left(Y_{i}-X_{i} B\right) .
$$

The residual error of observation is calculated as $V_{k}=$ $X_{k} B+\widehat{g}\left(t_{k}, B\right)-Y_{k}$, where $\widehat{g}\left(t_{k}, B\right)$ is expressed as $V=(I-$ $W)(X B-Y)$ in vector form, and then it is obtained from the least-square criterion:

$$
(X B-Y)^{T}(I-W)^{T} P(I-W)(X B-Y)=\min .
$$

The normal equation of the previous formula is

$$
X^{T}(I-W)^{T} P(I-W) X B=X^{T}(I-W)^{T} P(I-W) Y .
$$


If $X$ is of full rank, then $X^{T}(I-W)^{T} P(I-W) X$ is nonsingular. The least-square estimation of $B$ is

$$
\widehat{B}=\left(X^{T}(I-W)^{T} P(I-W) X\right)^{-1} X^{T}(I-W)^{T} P(I-W) Y .
$$

Substituting $\widehat{B}$ into the previous formula gets the estimation $\widehat{g}_{k}\left(t, \widehat{B}_{k}\right)$ of $g(t)$

$$
\widehat{g}_{k}\left(t, \widehat{B}_{k}\right)=\sum_{i=1}^{n} W_{i}(t)\left(L_{i}-X_{i} \widehat{B}_{k}\right)=W\left(Y-X \widehat{B}_{k}\right) .
$$

As analyzed previously and in consideration of the parametric statistical model, the semiparametric regression model can be expressed as

$$
K=K(H)+K(T)+K(\theta)+g(t)+\varepsilon,
$$

where $H$ is the water depth, $T$ is the temperature measurement value, $\theta$ is the time-related influence factor, $\varepsilon$ is the random error term, and $g(\cdot)$ is the part without clear function relation between the crack aperture and the parameter, which is called the nonparametric part of the model.

For the nonparametric part $g(\cdot)$, it is usually considered to be the systematic error or bias of the model in the general analysis. However, in the semiparametric model, it has a special meaning. Recent studies show [5-7] that concrete dam crack extension will occur if there is an unfavorable load, which means that its occurrence will lead to changes in the crack openings. According to the basic principles of semiparametric model, additional crack openings are generated due to the abnormality of the cracks' behavior, which largely constitutes the nonparametric part of this model. The nonparametric part of the semiparametric model reflects the abnormal characteristics of dam cracks to some extent, which provides a new idea in the abnormality diagnosis of these cracks' behavior.

2.2. The Validation of Semiparametric Statistical Model. For the regression analysis, if several nonstationary time series in the model are cointegrated, the regression analysis is still effective, and it is unnecessary to worry about problems caused by nonstationarity, such as spurious regression [26]. Take linear regression with variables $Y_{t}=\beta_{0}+\beta_{1} X_{t}+\varepsilon_{t}$ for example, due to $\varepsilon_{t}=Y_{t}-\beta_{0}-\beta_{1} X_{t}$, if $\left\{\varepsilon_{t}\right\}$ is smooth, then $\left\{Y_{t}-\beta_{0}-\beta_{1} X_{t}\right\}$ is also smooth, which means that either $Y_{t}$ and $X_{t}$ are stable themselves, or $Y_{t}$ and $X_{t}$ are single integrations with the same order and have a cointegration relationship. Regression analyses of models under these two cases are valid. Therefore, the model is effective as long as the error sequence is stable. As the stationarity of regression residuals sequence $\left\{e_{t}\right\}$ and error sequence remains consistent, the stability of $\left\{e_{t}\right\}$ can be tested by $\left\{\varepsilon_{t}\right\}$. Testing the cointegration among the time series can also test the smoothness of their linear regression residuals sequence. The idea of spurious regression and unit root in nonstationary time series compel us to examine the stationarity of regression residuals sequence.

In this paper, the unit root test method is used to analyze the stationarity of the semiparametric model residuals sequence, which is used for testing the validity of the semiparametric model. First, the regression model of the semiparametric model residuals sequence is established. Second, the characteristic equation of the regression model is extracted. Third, the characteristic roots of the characteristic equation are calculated. Finally, the distance between the characteristic roots and the unit circle are analyzed and compared. If all the characteristic roots are outside of the unit circle, the residual sequence is smooth. Otherwise, the residual sequence is unstable [31-33].

For the residuals sequence $\varepsilon_{1}, \varepsilon_{2}, \ldots, \varepsilon_{N}$ of the semiparametric model, the self-covariance function $\hat{v}_{k}$ can be expressed as

$$
\hat{v_{k}}=\frac{1}{N} \sum_{i=1}^{N-k}\left(\varepsilon_{i}-\mu\right)\left(\varepsilon_{i+k}-\mu\right), \quad k=0,1,2, \ldots, p,
$$

where, $\mu=(1 / N) \sum_{i=1}^{N} \varepsilon_{i}$.

Among them, the autocovariance function satisfies

$$
\left[\begin{array}{c}
v_{1} \\
v_{2} \\
\vdots \\
v_{p}
\end{array}\right]=\left[\begin{array}{cccc}
v_{0} & v_{1} & \ldots & v_{p-1} \\
v_{1} & v_{0} & \ldots & v_{p-2} \\
\vdots & \vdots & \ddots & \vdots \\
v_{p-1} & v_{p-2} & \ldots & v_{0}
\end{array}\right]\left[\begin{array}{c}
a_{1} \\
a_{2} \\
\vdots \\
a_{p}
\end{array}\right] .
$$

According to (13), the moment estimate $\hat{a_{1}}, \hat{a}_{2}, \ldots, \hat{a}_{p}$ of autoregressive coefficient can be obtained, and the moment estimate's formula of white noise variance is computed as follows:

$$
\begin{gathered}
{\left[\begin{array}{c}
\hat{a_{1}} \\
\hat{a_{2}} \\
\vdots \\
\hat{a_{p}}
\end{array}\right]=\left[\begin{array}{cccc}
\hat{v_{0}} & \hat{v_{1}} & \cdots & v_{p-1}^{\wedge} \\
\hat{v_{1}} & \hat{v_{0}} & \cdots & v_{p-2}^{\wedge} \\
\vdots & \vdots & \ddots & \vdots \\
\hat{v}_{p-1} & \hat{v}_{p-2} & \cdots & \hat{v_{0}}
\end{array}\right]^{-1}\left[\begin{array}{c}
\hat{v_{1}} \\
\hat{v_{2}} \\
\vdots \\
\hat{v_{p}}
\end{array}\right]} \\
\hat{\sigma}^{2}=\hat{v}_{0}-\sum_{i=1}^{p} \hat{a}_{i} \hat{v}_{i} .
\end{gathered}
$$

In view of the fact that the order autoregressive model is generally unknown, the order estimation is necessary. When the order is assumed as $k$, the corresponding variance $\hat{\sigma}_{k}^{2}$ of fitting residuals can be calculated. Moreover, by using $\hat{\sigma}_{k}^{2}$, the model order can be determined according to the QHIC criterion:

$\mathrm{QHIC}(k)$

$$
=\ln \left(\hat{\sigma}_{k}^{2}\right)+\frac{2 k \ln (\ln (n))}{n} \quad k=0,1,2, \ldots, p_{0},
$$

where $n$ is the residual number of samples and $p_{0}$ is the upper limit of the model order's value. When $\mathrm{QHIC}(k)$ is at minimum, the corresponding $k$ is called the QHIC order selection.

After the autoregressive model order and the coefficients of the model of crack residuals sequence are determined, 
the characteristic polynomial regression of the autoregressive model and its corresponding characteristic equation can both be obtained. If the autoregressive model residuals sequence of cracks is assumed, its corresponding characteristic equation is

$$
1-\sum_{j=1}^{p} a_{j} z^{j}=0
$$

The roots of the characteristic equation can be calculated according to formula (16). The minimum root of the characteristic equation is calculated as

$$
z_{\min }=\left|\min \left(z_{j}=\rho_{j} e^{i \lambda_{j}}\right)\right|,
$$

where $z_{j}=\rho_{j} e^{i \lambda_{j}}$ are all the roots of characteristic equation (16), including possible multiple roots, where $j=1,2, \ldots, p$ determine the closest distance between the root of the characteristic equation and the unit circle.

The closest distance between the root of the characteristic equation and the unit circle must be determined. If $z_{\min }>$ 1 , the residuals sequence is smooth and the semiparametric model is proved to be effective; then we model and analyze the cracks data sequence using the semiparametric model.

\section{Project Case Analysis}

The crack near the elevation of $105 \mathrm{~m}$ at the lower stream of a gravity arch dam is analyzed based on the semiparametric model. This dam is a concrete gravity dam of concentric circle with variable radiuses. The dam's top elevation is $126.3 \mathrm{~m}$, its maximum height is $76.3 \mathrm{~m}$, and it has 28 sections from left to right. The construction of the dam took 12 years, which was executed in three phases. During the casting of concrete in Phase II, the layer was raised quickly and the interval time of casting the layer was short. The shrinkage distortion of concrete in Phase II was strongly constrained by the concrete in Phase I, causing a crack at the top, which extended from dam block number 5 to number 28, with a length of over $300 \mathrm{~m}$. Upon detection, the crack was up to $5 \mathrm{~m}$ deep. Due to this, a cross-dam reinforcing grouting treatment was conducted from dam block number 14 to number 20 in 1973. In 1987, the crack was re-treated with epoxy grouting. The 1680 crack mouth opening displacement data monitored by the crack meter embedded in dam block number 18 from October 10, 1974 to December 18, 2006 were analyzed. According to the parametric statistical model, the CMOD statistical model form of the crack aperture can be expressed as

$$
\mathrm{CMOD}=\sum_{i=0}^{m_{1}} a_{i} H^{i}+\sum_{i=1}^{m_{2}} b_{i} T_{i}+\left(c_{1} \theta+c_{2} \ln \theta\right)+\varepsilon .
$$

According to the semiparametric regression model, the CMOD semiparametric regression model of the crack aperture can be expressed as

$$
\mathrm{CMOD}=\sum_{i=0}^{m_{1}} a_{i} H^{i}+\sum_{i=1}^{m_{2}} b_{i} T_{i}+\left(c_{1} \theta+c_{2} \ln \theta\right)+g(t)+\varepsilon .
$$

The basic idea of the regression model is to use the explaining variable included in the model to explain the change in the dependent variable as extensive as possible. Without a reference standard or a guiding policy, it cannot be determined if the model that is selected for the empirical analysis is proper. Generally, a good model should meet the following standards: time saving, identifiable, goodness-offit, and consistency in its theoretical and forecasting abilities [34]. The fitting degree of goodness-of-fit can be measured by the calibrated sample determination coefficient $R^{2}$ and $F$ values, which are defined as $R^{2}=$ ESS/TSS; $F=\left[R^{2} /(k-\right.$ $1)] /\left[\left(1-R^{2}\right) /(n-k)\right]$, where ESS is the regression sum of squares, TSS is the total sum of squares, $n$ is the sample capacity, and $k$ is the explaining variable and the intercept. It can be seen from the definition of $R^{2}$ that the closer $R^{2}$ is to 1 , the better the resulting regression fitting will be. It can be understood from the definition of $F$ that $F$ and $R^{2}$ change in the same direction. In other words, the higher the values of $F$ and $R^{2}$ are, the better the model fitting will be.

By applying the Akaike information standard or AIC, and the Schwarz information standard or SIC, the forecast effect of the regression model can be tested. These two information standards are defined as AIC $=\exp (2 k / n)\left(\sum e_{t}^{2}\right) / n$ and SIC $=$ $n^{k / n}\left(\sum e_{t}^{2}\right) / n$, in which $n$ is the sample capacity, $k$ is the number of variables in the model, and $\sum e_{t}^{2}$ is the residual sum of squares of the sample. Based on the definitions of AIC and SIC, the previous factors can be deemed as penalty factors to the degree of freedom. The more explaining variables are in the model, the heavier the penalty will be. Relatively, the SIC standard has a heavier penalty than the AIC standard to the degree of freedom. For AIC and SIC, in terms of forecast, the lower the measuring value is, the better the model's forecast will be.

Efficiency, fairness, and consistency are then tested. Efficiency is the ratio between the forecasted value and actual value, so the more the evaluation ratio is approximate to 1 , the higher the efficiency will be. Fairness is weighed with the coefficient of variation, which is the ratio between the standard deviation and mean value. For the evaluation of different samples, the evaluation ratio should be the same as much as possible. Consistency means that the evaluation ratio of different samples should not generate progression or regression and that the evaluation result should be consistent with the actual value [35].

According to the solution method of the parametric statistical model, the measured and fitted CMOD are shown in Figure 1, and the residual CMOD curve is shown in Figure 2. Similarly, for the semiparametric statistical model, the measured and fitted CMOD curves are shown in Figure 3, and the residual CMOD curve is shown in Figure 4. See Figures 5 and 3 for the parametric and nonparametric CMOD curves, and see Table 2 for the values of regression and fitted goodness coefficients.

It can be seen that (1) in the regression fitting effect to the original crack aperture, the fitted goodness parameters $F$ and $R$ of the semiparametric regression model are $1.256 E+04$ and 0.991, respectively, while those of the parametric regression model (Table 1) are $1.448 E+03$ and 0.926 , respectively. Thus, 
TABLE 1: Coefficients of the regression and the fitted goodness (parametric model).

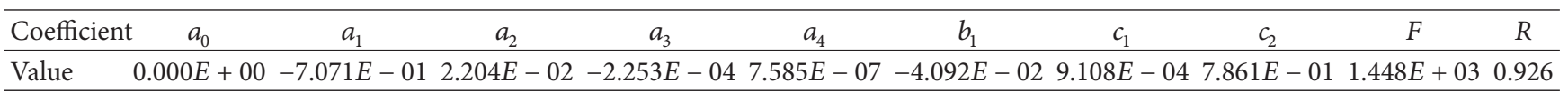

TABLE 2: Coefficients of the regression and the fitted goodness (semiparametric model).

\begin{tabular}{|c|c|c|c|c|c|c|c|}
\hline Coefficient & $a_{0}$ & $a_{1}$ & $a_{2}$ & $a_{4}$ & $c_{2}$ & $F$ & $R$ \\
\hline Value & $0.000 E+00$ & $-6.505 E-01$ & $2.032 E-02-2.080 E-04$ & $7.001 E-07-3.781 E-02$ & $8.068 E-047.911 E-01$ & $1.256 E+04$ & 0.991 \\
\hline
\end{tabular}

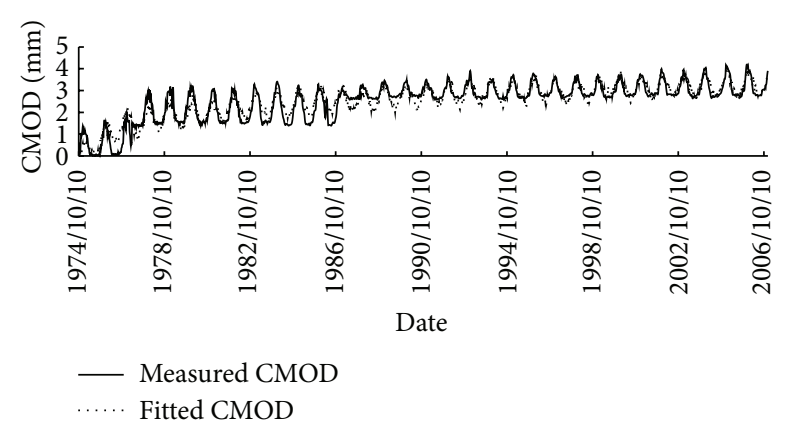

FIGURE 1: Comparison curves of measured and fitted CMOD (parametric model).

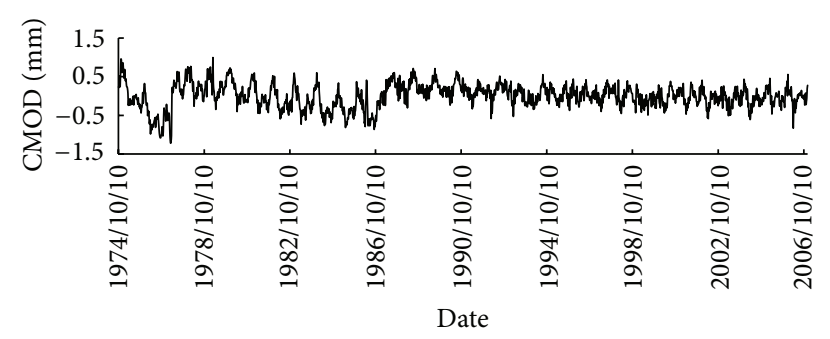

FIgURE 2: Residual CMOD curves (parametric model).

the semiparametric model is superior to the parametric regression model; (2) in the characteristics of the parametric sequence of crack aperture, the residual process of the parametric regression model clearly indicates that the residual error does not display a random model, but a certain system model, that is, the error presents systematicness in size and symbol, in Figure 2, it presents a relatively strong periodicity. While the randomness of residual process of the semiparametric regression model is relatively significant, it can be seen that the semiparametric regression model has a stronger explaining ability than the parametric regression model to the original crack aperture.

In order to test the validity of the semiparametric statistical model in the safety monitoring of concrete dam cracks, the residual sequence is segmented and the characteristic roots of each period are calculated. As shown in Figure 7, all minimum eigenvalues of each period are greater than 1.0, indicating that the semiparametric model residuals sequence is stationary; that is, the semiparametric model satisfies the test of validity, the regression analysis on crack sequence can be conducted by using the semiparametric model, and the related forecast monitoring can be carried out.

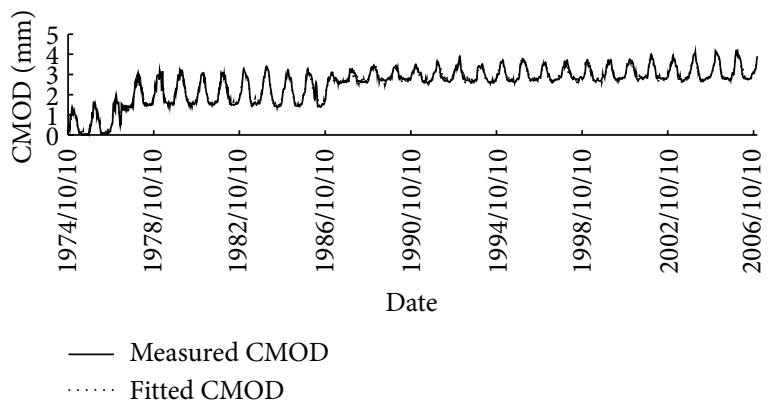

FIGURE 3: Comparison curves of measured and fitted CMOD (semiparametric model).

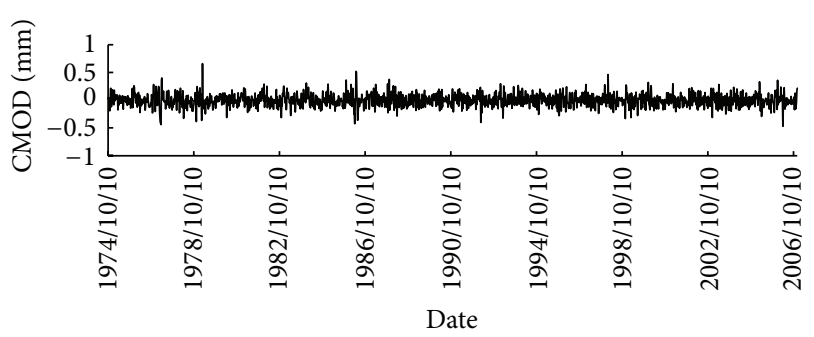

FIgURE 4: Residual CMOD curves (semiparametric model).

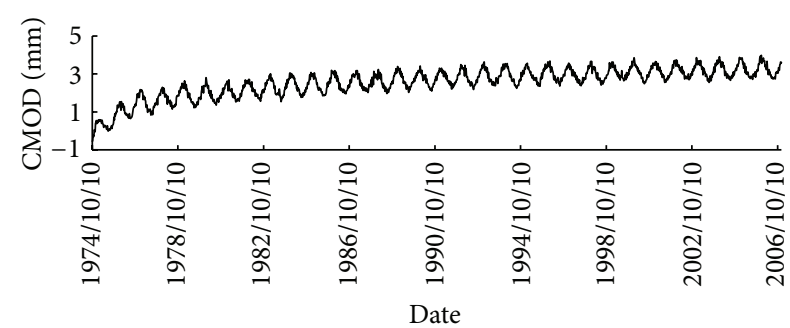

FIGURE 5: Parametric component curves of the CMOD (semiparametric model).

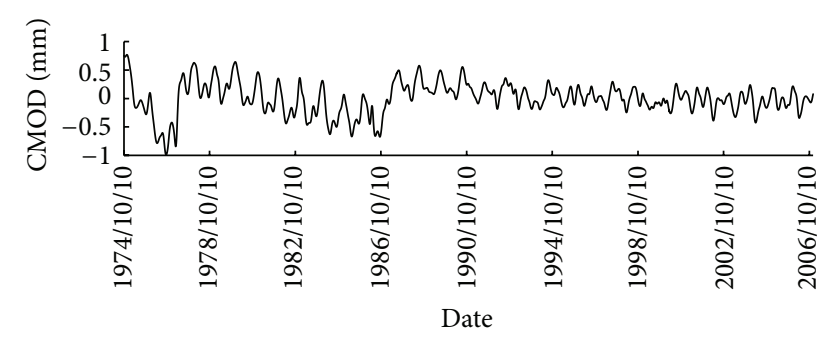

FIgure 6: Nonparametric component curves of the CMOD (semiparametric model). 
TABLE 3: Predicting outcomes of statistical model and Semiparametric regression model.

\begin{tabular}{|c|c|c|c|c|c|c|c|c|c|}
\hline \multirow{2}{*}{ Date } & \multirow[b]{2}{*}{$\begin{array}{l}\text { Measured } \\
\text { CMOD (mm) }\end{array}$} & \multicolumn{8}{|c|}{ Predicted CMOD $(\mathrm{mm})$} \\
\hline & & $\begin{array}{l}\text { Semiparametric } \\
\text { regression } \\
\text { model }\end{array}$ & $\begin{array}{c}\text { Parametric } \\
\text { statistical } \\
\text { model [28] }\end{array}$ & $\begin{array}{c}\text { uncertainty } \\
\text { model [8] }\end{array}$ & $\begin{array}{l}\text { Improved } \\
\text { parametric } \\
\text { statistical } \\
\text { model [2] }\end{array}$ & $\begin{array}{c}\text { Abnormality } \\
\text { monitoring } \\
\text { model [9] }\end{array}$ & $\begin{array}{c}\text { Hybrid } \\
\text { prediction } \\
\text { model of } \\
\text { statistics } \\
\text { and chaos } \\
{[10]}\end{array}$ & $\begin{array}{c}\text { Monitoring } \\
\text { model with } \\
\text { RS-BP } \\
\text { blending } \\
\text { [11] }\end{array}$ & $\begin{array}{c}\text { Hybrid model } \\
\text { based on XFEM } \\
\text { (extended finite } \\
\text { element } \\
\text { method) [12] }\end{array}$ \\
\hline $2005-1-1$ & 4.17 & 3.92 & 4.03 & 4.25 & 4.06 & 3.97 & 3.89 & 4.10 & 4.32 \\
\hline $2005-6-16$ & 2.82 & 3.11 & 3.12 & 2.63 & 2.73 & 2.69 & 2.97 & 2.80 & 2.96 \\
\hline $2005-10-20$ & 3.09 & 3.21 & 2.97 & 3.25 & 3.23 & 2.86 & 2.97 & 3.23 & 2.79 \\
\hline $2006-3-16$ & 3.48 & 3.36 & 3.16 & 3.17 & 3.38 & 3.26 & 3.53 & 3.60 & 3.31 \\
\hline $2006-6-8$ & 2.79 & 2.96 & 2.53 & 2.56 & 2.68 & 2.81 & 2.91 & 2.59 & 2.63 \\
\hline $2006-9-1$ & 2.83 & 2.91 & 2.68 & 2.79 & 2.96 & 2.79 & 2.91 & 2.69 & 2.81 \\
\hline 2006-11-9 & 3.25 & 3.35 & 3.42 & 3.08 & 3.37 & 3.16 & 3.29 & 3.17 & 3.32 \\
\hline 2006-12-14 & 3.90 & 3.97 & 4.11 & 4.12 & 3.78 & 3.82 & 4.03 & 3.89 & 4.06 \\
\hline \multicolumn{2}{|c|}{ Information standards AIC } & 0.057 & 0.052 & 0.053 & 0.055 & 0.056 & 0.054 & 0.053 & 0.058 \\
\hline \multicolumn{2}{|c|}{ Information standards SIC } & 0.070 & 0.064 & 0.068 & 0.069 & 0.071 & 0.069 & 0.067 & 0.072 \\
\hline
\end{tabular}

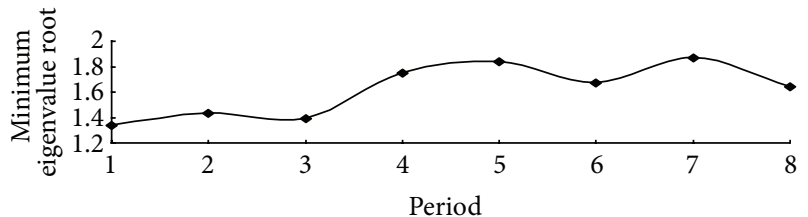

FIGURE 7: The curve of the minimum eigenvalue root in each period.

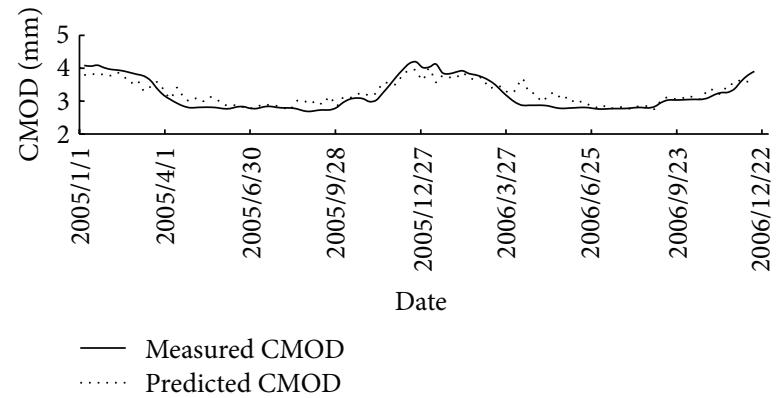

FIgURE 8: The prediction curve of the CMOD (semiparametric model).

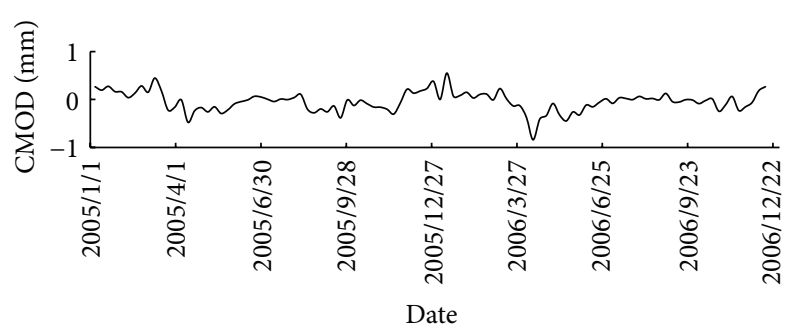

FIGURE 9: Residual curve of the predicted CMOD (semiparametric model).
In order to analyze the forecast effect of the semiparametric statistical model, select crack openings in the time period from 2005-1-1 to 2006-12-22 were used as predictive samples. The measured values are compared and predicted (see Figure 8), and the process curve of prediction error sequence is shown in Figure 9. Meanwhile, the predicted outcomes of the semiparametric model in typical moments are shown in Table 3. We can see from Figures 8 and 9 and Table 3 that AIC and SIC amounted to 0.055 and 0.070 , respectively, indicating that the forecast effect of these models is good. In particular, for the parametric regression model, its information standards are, respectively, at 0.052 and 0.064 , while the information standards of the semiparametric regression models are, respectively, at 0.057 and 0.070 . The semiparametric regression model is used for forecast, but the forecast is only based on the parameters, so the forecast effect of the model is relatively poor. However, the information standards of the two models are approximate. It can be seen that the forecast of the parametric regression model is equivalent to that of the semiparametric statistical model without significant difference.

\section{Conclusion}

(1) Cracks are one of the hidden dangers in concrete dams, and studying the safety monitoring model of concrete dam cracks has always been a hot topic in such field. The current crack safety monitoring model is the parametric statistical model, which is implemented without fully considering the nonlinear relation between each factor and the irreversible deformation that is caused by hydraulic pressure and temperature loads. The residual error contains plenty of information on both concrete development and evolution.

(2) On the basis of the parametric statistical model and by introducing the nonparametric component of concrete dam 
aperture to explain the parts that are imperfectly explained by the parametric model, the semiparametric statistical model of safety monitoring of cracks in concrete dams is established to consider linearity-related factors and to overcome the limitation of the parametric model in expressing the objective model, making this statistical model more appropriate for the objective practice and enabling it to separate system error and accidental error from the error, providing richer calculations for the safety monitoring of cracks in concrete dams.

(3) The semiparametric statistical model is applied in a project, and the result shows that the fitted goodness of the semiparametric statistical model is superior to that of the parametric statistical model. Meanwhile, in the characteristics of the parametric sequence of crack aperture, the residual curve of the parametric statistical model clearly shows that the residual error does not show a random model but a certain system model, while the randomness of the residual curve of the semiparametric statistical model is relatively significant; that is, the semiparametric statistical model has a stronger explaining ability of the original crack aperture than the parametric statistical model. In terms of forecast ability, when the forecast is only based on the parameter of the nonparametric statistical model, its forecast effect is equivalent to that of the parametric statistical model without showing any significant difference.

(4) The semiparametric statistical model is modeled on a simple and reasonable principle and a strong practice, laying a theoretical foundation for the safety monitoring of cracks in concrete dams, with good application prospects in the actual project.

(5) In this paper, studies of the semiparametric statistical model in crack mouth opening displacement have been conducted tentatively, but there are still many issues that need to be studied, such as the following. (1) the parametric section of the semiparametric model is used in predicting crack mouth opening displacement in this paper, and its predictions are the same with the predictions of the parametric statistical model. Therefore, the method of overall prediction using the semiparametric model can become an important research subject in the future; (2) based on this paper's findings, the semiparametric statistical model has a better regression effect in analyzing crack mouth opening displacement. To some extent, the state of abnormality in cracks behavior is reflected in the nonparametric part of the semiparametric model. Thus, the study of the abnormality problem in cracks behavior based on the semiparametric model will also become an important research subject in the future.

\section{Acknowledgments}

This work was supported by Program Sponsored for Scientific Innovation Research of College Graduate in Jiangsu province (Grant no. CXZZ12_0248), the Program for New Century Excellent Talents in University (Grants nos. NCET-11-0628, NCET-10-0359), the Open Foundation of State Key Laboratory of Hydrology-Water Resources (Grant no. 2012490211), the Fundamental Research Funds for the Central Universities (Grant no. 2012BSJJ007), and the Key Project of the National Natural Science Foundation of China (Grant no. 51139001).

\section{References}

[1] ICOLD, World Register of Dams, International Commission on Large Dams, Paris, France, 1988.

[2] Z. Li, J. Wang, T. Bao, and H. Hou, "Improved safety monitoring model of crack in concrete dams," in Proceedings of the 12th International Conference on Engineering, Science, Construction, and Operations in Challenging Environments-Earth and Space, pp. 559-566, ASCE, March 2010.

[3] Z. Wu and C. Gu, Safety Monitoring Theories of Hydraulic Structures and Their Application, Hihger Education Process, Beijing, China, 2003.

[4] T. Bao, Chaotic Characteristics of Cracks in Concrete Dam and Their Analysis Theory and Mehtod, Hohai University, Nanjing, China, 2004.

[5] X. Li, H. Xu, C. Gu, and Z. Wu, "Detecting abnormality point of dam crack based on phase plane," Journal of Basic Science and Engineering, vol. 15, no. 2, pp. 177-182, 2007.

[6] T. Bao and H. Yu, "Detection of subcritical crack propagation for concrete dams," Science in China, Series E: Technological Sciences, vol. 52, no. 12, pp. 3654-3660, 2009.

[7] C. Gu, Z. Li, and B. Xu, "Abnormality diagnosis of cracks in the concrete dam based on dynamical structure mutation," Science China Technological Sciences, vol. 54, no. 7, pp. 1930-1939, 2011.

[8] F. J. Chen, T. Na, and E. F. Zhao, "Uncertainty analysis model of deformation of cracks in concrete dam," Journal of Heilongjiang Hydraulic Engineering Collee, vol. 36, no. 1, pp. 54-60, 2009.

[9] T. Bao, D. Qin, X. Zhou, and G. Wu, "Abnormality monitoring model of cracks in concrete dams," Science China Technological Sciences, vol. 54, no. 7, pp. 1914-1922, 2011.

[10] T. Bao and Z. Wu, "Hybrid prediction model of concrete crack openings," Water Resources and Hydropower Engineering, 2005.

[11] P. Lei and F. Xiao, "Crack monitoring model based on RS-BP blending," Journal of Changsha University of Science and Technology (Natural Science Edition), vol. 8, no. 2, pp. 45-50, 2011.

[12] D. J. Zheng, Z. Y. Huo, and B. Li, "Arch-dam crack deformation monitoring hybrid model based on XFEM," Science China Technological Sciences, vol. 54, no. 10, pp. 2611-2617, 2011.

[13] P. J. Green and B. W. Silverman, Nonparametric Regression and Generalized Linear Models. A Roughness Penalty Approach, vol. 58 of Monographs on Statistics and Applied Probability, Chapman \& Hall, London, UK, 1994.

[14] R. L. Eubank, J. D. Hart, and P. Speckman, “Trigonometric series regression estimators with an application to partially linear models," Journal of Multivariate Analysis, vol. 32, no. 1, pp. 7083, 1990.

[15] A. V. Ivanov and N. N. Leonenko, "Semiparametric analysis of long-range dependence in nonlinear regression," Journal of Statistical Planning and Inference, pp. 1733-1753, 2008.

[16] Y. C. Shi and G. X. Chai, "Local polynomial smoothing of semiparametric regression model," Journal of Tongji University, vol. 28, no. 1, pp. 80-83, 2000.

[17] L. R. Zen, Statistical Diagnosis of Semi-Parametric Generalized Linear Models, East China Normal University, Shanghai, China, 2004.

[18] H. L. Hu, "Strong consistency of wavelet estimation in semiparametric regression model," Chinese Version of Mathematics, vol. 49, no. 6, pp. 1418-1424, 2006.

[19] D. Ruppert, M. P. Wand, and R. J. Carroll, Semiparametric Regression, vol. 12 of Cambridge Series in Statistical and Probabilistic Mathematics, Cambridge University Press, Cambridge, UK, 2003. 
[20] S. Wang and C. Y. Wang, "A note on kernel assisted estimators in missing covariate regression," Statistics \& Probability Letters, vol. 55, no. 4, pp. 439-449, 2001.

[21] S.-Y. Hong, "Normal approximation rate and bias reduction for data-driven kernel smoothing estimator in a semiparametric regression model," Journal of Multivariate Analysis, vol. 80, no. 1, pp. 1-20, 2002.

[22] P. M. Robinson and M. Henry, "Higher-order kernel semiparametric $M$-estimation of long memory," Journal of Econometrics, vol. 114, no. 1, pp. 1-27, 2003.

[23] S. Manzan and D. Zerom, "Kernel estimation of a partially linear additive model," Statistics \& Probability Letters, vol. 72, no. 4, pp. 313-322, 2005.

[24] N. L. Hjort and S. G. Walker, "A note on kernel density estimators with optimal bandwidths," Statistics and Probability Letters, vol. 54, no. 2, pp. 153-159, 2001.

[25] M. Hagmann and O. Scaillet, "Local multiplicative bias correction for asymmetric kernel density estimators," Journal of Econometrics, vol. 141, no. 1, pp. 213-249, 2007.

[26] W. Song, "Moderate deviations for deconvolution kernel density estimators with ordinary smooth measurement errors," Statistics \& Probability Letters, vol. 80, no. 3-4, pp. 169-176, 2010.

[27] C. Xu, D.-J. Yue, Y.-F. Dong, and C.-F. Deng, "Regression model for dam deformation based on principal component and semiparametric analysis," Yantu Lixue/Rock and Soil Mechanics, vol. 32, no. 12, pp. 3738-3742, 2011.

[28] C. S. Gu and Z. R. Wu, Safety Monitoring Theories and Methods of Dam and Dam Foundation and Theri Application, Hohai University Press, Nanjing, China, 2006.

[29] A. A. Wells, "Applications of fracture mechanics atand beyond general yielding," British Welding Journal, vol. 10, pp. 563-570, 1963.

[30] S. L. Zhang, Study on Theory and Application of Least Square Estimation of Nonlinear Semi-Parametric Model, Wuhan University, Wuhan, China, 2003.

[31] F. Q. Li, Analysis Method of Dam Safety Monitoring Data, Zhejiang University, Hangzhou, China, 2012.

[32] J. Qian, F. Q. Li, and Y. Zhang, "A study on dam deformation monitoring index based on wavelet transformation and characteristic polynomial root of an auto-regression model," China Civil Engineering Journal, vol. 42, no. 6, pp. 140-144, 2009.

[33] F. Q. Li and J. Qian, "Application of characteristic polynomial roots of autoregression time-series model in analysis of dam observation data," Journal of Zhejiang University, vol. 43, no. 1, pp. 193-196, 2009.

[34] G. Lati, Econometrics, Renmin University Press, Beijing, China, 2000.

[35] J. Jikun, Study on Application of Nonparametric Regression in the Hedonic Price Evaluation Method of Commercial Residential Buildings, Zhejiang University, Hangzhou, China, 2006. 


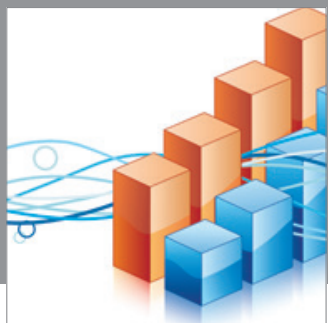

Advances in

Operations Research

mansans

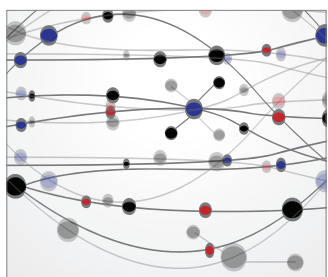

The Scientific World Journal
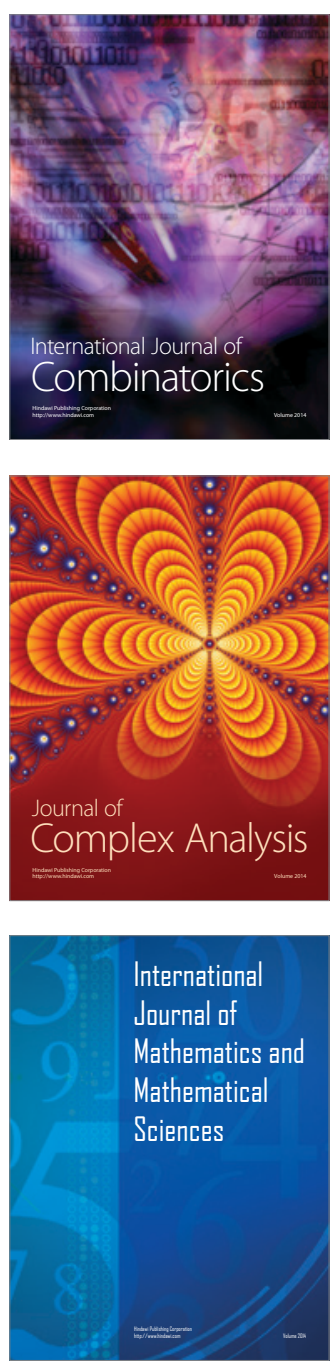
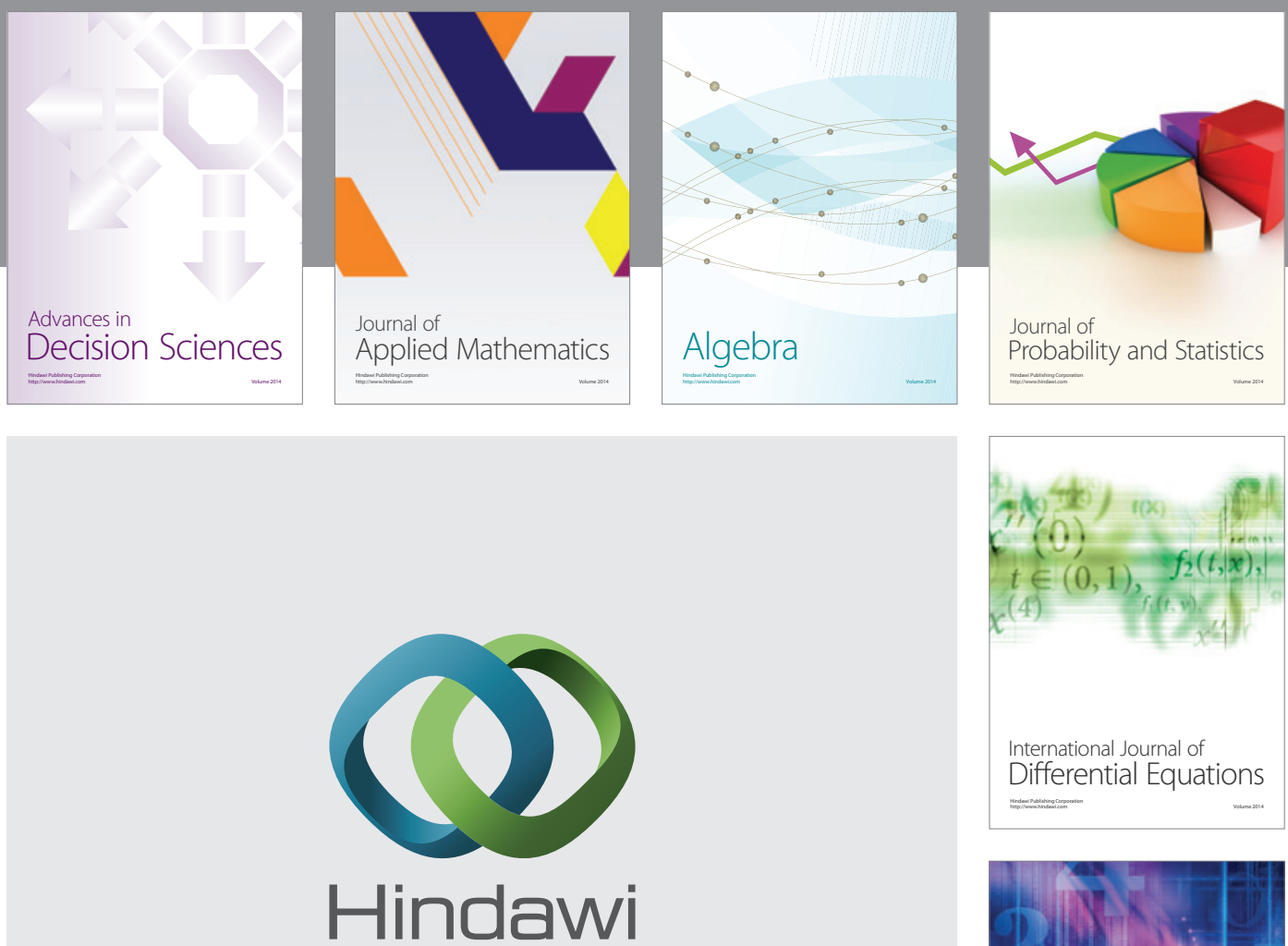

Submit your manuscripts at http://www.hindawi.com
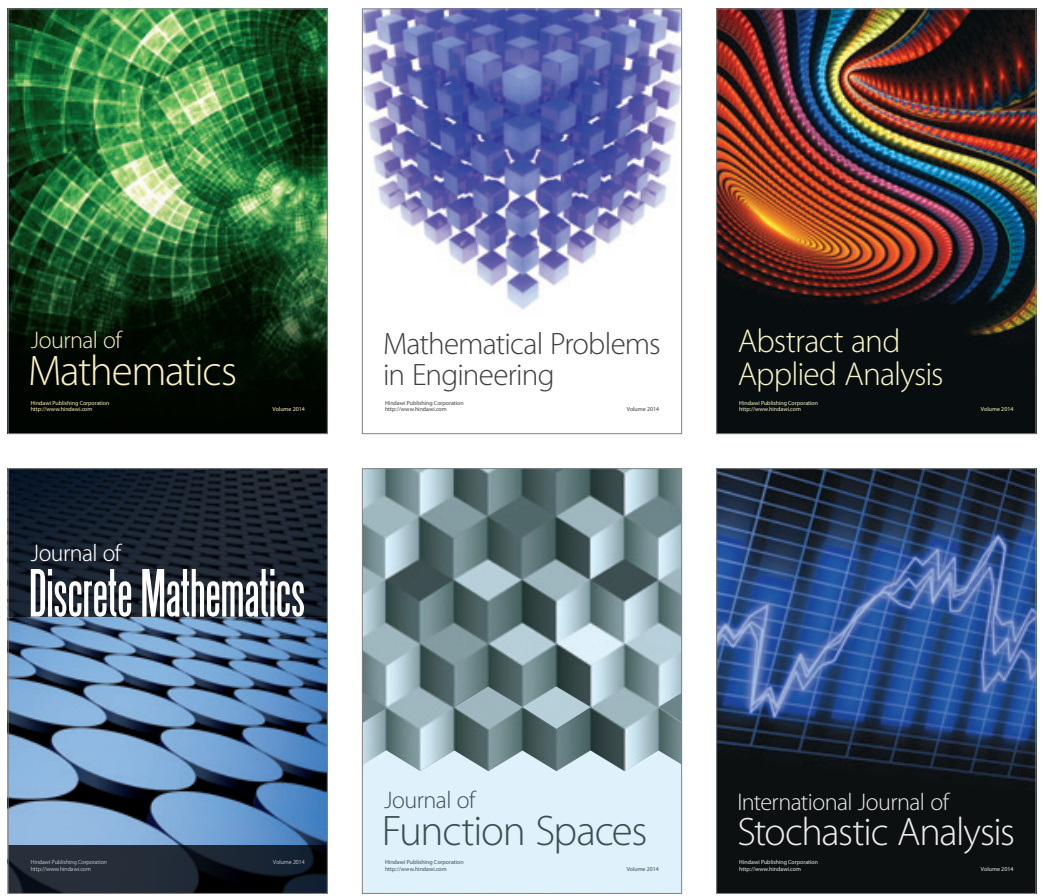

Journal of

Function Spaces

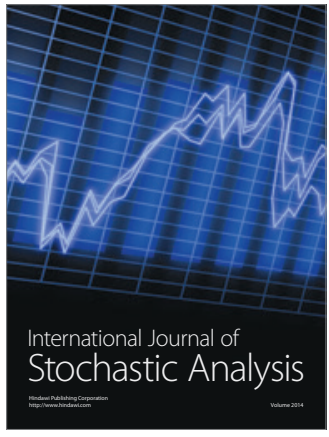

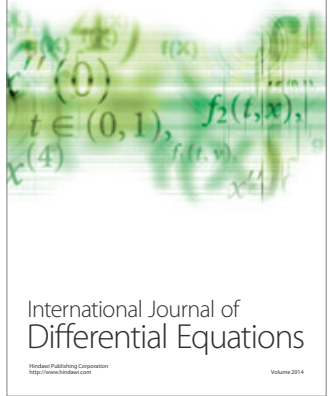
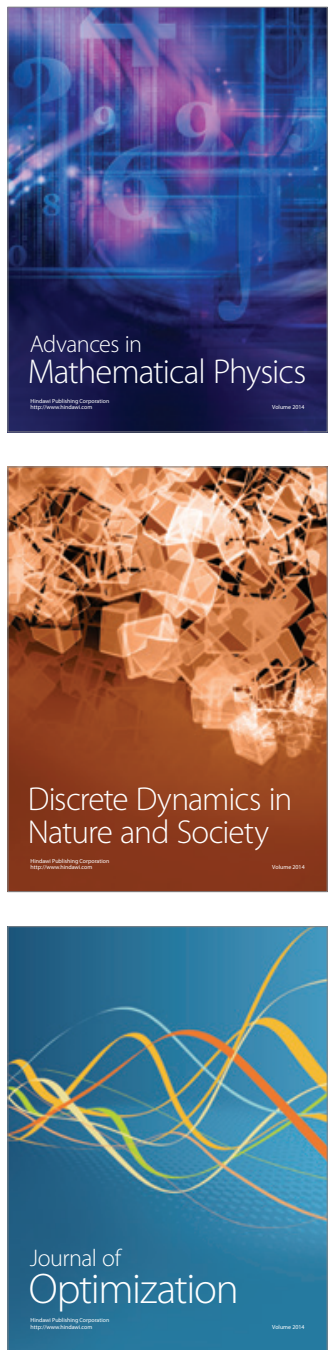